\title{
Sociología y opinión pública: desmontando un dispositivo
}

\author{
Juan Pablo Venables Brito ${ }^{1}$
}

Fecha de recepción: 9 de noviembre de 2017

Fecha de aprobación: 22 de enero de 2018

\begin{abstract}
Resumen
El presente artículo parte de la hipótesis que afirma que se ha extendido con éxito la idea de que la ocupación central de la sociología es realizar encuestas de opinión pública, y que esta idea se habría convertido en un dispositivo de saber/poder, en términos de Foucault. El objetivo central del artículo es desmontar dicho dispositivo, mediante el análisis tanto de sus falencias como de sus carencias. Para las falencias, se lleva adelante un análisis histórico-conceptual del concepto de "opinión pública" y de la industria que se ha desarrollado en torno a ella, deteniéndose en sus presupuestos epistemológicos y metodológicos, haciendo uso de ejemplos actuales de encuestas de opinión realizadas en Chile y en el extranjero. Para el análisis de las carencias, por su parte, se propone una comprensión de la actividad sociológica como una ciencia inherentemente explicativa y no reductible a método alguno. Una vez desmontado el dispositivo, se concluye que no es posible concebir la medición de la opinión pública mediante encuestas como práctica sociológica.
\end{abstract}

Palabras clave: opinión pública, dispositivo, sociología, encuestas.

\section{Sociology and public opinion: Dismantling a device}

\begin{abstract}
This article is based on the hypothesis about the successful extension of the idea that suggests the main task of sociology is conducting public opinion surveys, and that this idea would have become a knowledge/power device, in Foucault's terms. The main objective of this article is to dismantle such a device by analyzing both its shortcomings and deficiencies. For shortcomings, it carries out a historical-conceptual analysis about the "public opinion" concept and the industry developed around, noting its epistemological and methodological assumptions, making use of current examples of opinion surveys carried out in Chile and abroad. For the analysis of deficiencies, on the other hand, it proposes to understand the sociological activity as an inherently explanatory science not reducible to any method. Once the device
\end{abstract}

Sociólogo, Magíster en Filosofía y $\operatorname{Dr}(\mathrm{c})$ en Ciencias Sociales. Universidad Austral de Chile, Valdivia, Chile. Contacto: pvenables@uach.cl. 
has been dismantled, it concludes that it is not possible to measure the public opinion through surveys as a sociological practice.

Keywords: Public opinion, device, sociology, surveys.

\title{
Sociologia e opinião pública: desmontando um dispositivo
}

\begin{abstract}
Resumo
O presente artigo parte da hipótese que afirma que se tem estendido com sucesso a ideia de que a ocupação central da sociologia é realizar sondagens de opinião pública, e que esta ideia teria se tornado um dispositivo de saber/poder, em termos de Foucault. O objetivo central do artigo é desmontar dito dispositivo através da análise tanto das suas falências quanto das suas carências. Para as falências, desenvolve-se uma análise histórico-conceptual do conceito de "opinião pública" e da indústria que se tem desenvolvido em torno dela, detendo-se nos seus pressupostos epistemológicos e metodológicos, fazendo uso de exemplos atuais de sondagens de opinião realizadas no Chile e no exterior. Para a análise das carências, por sua vez, propõe-se uma compreensão da atividade sociológica como uma ciência inerentemente explicativa e não redutível a método algum. Uma vez desmontado o dispositivo, conclui-se que não é possível conceber a medição da opinião pública através de sondagens como prática sociológica.
\end{abstract}

Palavras-chave: opinião pública, dispositivo, sociologia, sondagens.

\section{Introducción}

Este artículo se basa en la hipótesis de que se ha extendido con éxito la idea de que la ocupación central de la sociología es realizar encuestas de opinión pública, y de que esta idea se habría convertido en un dispositivo de saber/poder, en términos de Foucault.

Este dispositivo representa, por un lado, una reducción del quehacer sociológico a la medición de la opinión mediante encuestas, pero, más importante aún, limita el papel inherentemente explicativo y crítico de la disciplina sociológica, contribuyendo con ello a la generación de una pseudociencia dedicada a medir la opinión, sobre el entendido de que ésta sería científica, verdadera y democrática, escondiendo así la falacia metodológica que le subyace y las carencias metodológicas, epistemológicas, ontológicas y teóricas que implica. El objetivo central del artículo es, entonces, desmontar dicho dispositivo. 
El artículo está dividido en dos partes. La primera es una crítica a la noción de "opinión pública" y a la reducción de la sociología a su medición; la segunda pretende expandir el quehacer sociológico más allá de las fronteras de la opinión, de modo de desmontar el dispositivo, tanto por sus falencias como por sus carencias.

La primera parte, que incluye las secciones 1 a 5, está destinada a la revisión histórica de la industria de la "opinión pública" -con especial énfasis en el caso de Chile- y a la relación que dicho concepto ha establecido con la sociología, de modo de evidenciar las bases del dispositivo. Asimismo, se sientan las bases teóricoconceptuales de la noción de "dispositivo" desde Foucault, lo que entrega luces analíticas para el artículo. Al mismo tiempo, se desarrollan las principales críticas a las encuestas de opinión y su pretensión de verdad, cientificidad y democraticidad, así como a la falacia de la neutralidad metodológica que le subyace. Con esto se busca dar cuenta de que la opinión pública se ha convertido en una suerte de fetichización mercantilizada de la creencia, a través de una creciente industria de la opinión que, más que medir un fenómeno social existente, confecciona un artefacto en el devenir de su proceso productivo; en otras palabras: produce opinión. Esto se complementa con ejemplos actualizados de encuestas, en las que se evidencia la falacia de neutralidad metodológica.

La segunda parte se compone de la sección seis y está destinada a exponer brevemente algunas propuestas teóricas que "combaten" este dispositivo, al concebir el quehacer sociológico como eminentemente explicativo. Aquí se destaca el planteamiento de Archer (2009) en torno al conflacionismo ascendente y, especialmente, la necesidad de vincular los niveles ontológicos, teóricos y metodológicos en la investigación sociológica.

\section{La medición de la opinión pública como una industria y sus actores}

El nacimiento de la industria de la opinión pública suele fijarse en 1935, en Estados Unidos, con la fundación del Instituto 
Americano de Opinión Pública, por George Gallup; instituto que, al año siguiente, se transformaría en la exitosa y vigente empresa Gallup, dedicada a la medición de audiencias y encuestas de opinión, cuyo éxito seminal fue predecir el resultado de una elección presidencial utilizando una muestra de solo cinco mil personas (Cordero y Tapia, 2007).

En términos técnicos, el impacto principal de Gallup al predecir el resultado de la elección presidencial de 1936 se basó en dos cuestiones metodológicas clave: el desarrollo de escalas de medición de actitudes -cuyo creador fue el psicólogo L. Thurstone en 1928- y el desarrollo de técnicas de muestreo, cuyo pionero fue Gallup, en 1936.

El éxito de Gallup significó el puntapié inicial para el desarrollo de una industria de la opinión pública, que ha crecido exponencialmente y se ha extendido por todo el orbe. Ese desarrollo se explicaría por cuatro razones principales vinculadas entre sí: en primer lugar, por el comienzo del predominio de una visión de la opinión pública como un activo previsible, posible de ser conocido y medido; en segundo lugar porque, dada su previsibilidad, se vuelve un producto irresistible y casi irrecusable para los intereses políticos; en tercero porque, en tanto instrumento de medición de la opinión de los individuos, su utilidad se hace extensiva a los intereses del mercado (Garrigou, 2007), y en cuarto lugar porque crea un producto interpretable como cualquier otra mercancía (Marx, 1973) y que no existía con anterioridad: la opinión. O, como señala Bourdieu (2002), porque la finalidad de estas mediciones no es conocer la opinión pública sino más bien producirla.

En el caso de Chile, el inicio formal de la industria de medición de la opinión pública data de 1957, fecha en que el sociólogo Eduardo Hamuy inició un programa de encuestas de opinión desde el Departamento de Sociología de la Universidad de Chile, que se extendió exitosamente hasta el golpe de Estado de 1973. Sin embargo: 
No es sino hasta el plebiscito de 1988 que las encuestas experimentan una notable expansión e importancia en la vida pública, situación que se acentúa sobre todo desde la elección presidencial del año 1999 cuando se instala definitivamente la comunicación estratégica y el marketing en la actividad política. (Cordero y Tapia, 2007, p. 6)

A modo de breve reconstrucción histórica nacional, en 1975 se crea Adimark, seguida por Cadem en 1976, ICCOM en 1977, Time en 1979 y Demoscópica en $1982^{2}$ (Cordero y Tapia, 2007). La mayoría de ellas se desarrollan como empresas dedicadas tanto al sondeo de la opinión pública como a la investigación de mercado. A dichas empresas locales se suman las filiales nacionales de empresas extranjeras, como Gallup en 1975, seguida luego por IPSOS y MORI en 1994.

El claro peso político que adquiere el manejo de este tipo de información, sumado a la coyuntura sociopolítica del plebiscito de 1988, que ganó la opción No, marcando la salida de Pinochet, abre la industria a grupos de interés ideológico congregados en la figura de centros de estudios, como el Centro de Estudios Públicos (CEP), ligado a la derecha, o el Centro de Estudios de la Realidad Contemporánea (CERC), fundado en 1986 y ligado a la Democracia Cristiana. En esta misma lógica, aunque de manera más reciente, se suman a la industria de medición de la opinión pública fundaciones políticas y think tanks, como el Instituto Libertad y Desarrollo, la Fundación Futuro, la Fundación Chile 21, entre otros.

Las universidades, por su parte, también se incorporan de manera reciente a la industria. Luego del golpe de Estado de 1973, la Universidad de Chile es conminada por la dictadura a desistir de sus mediciones. Así, la vuelta de las universidades en

Estas fechas son tomadas del artículo de Cordero y Tapia (2007). Sin embargo, debe señalarse que tanto Adimark como Cadem indican en sus páginas web que su fecha de creación es 1974. 
los 90 se produce en el contexto de una industria de la opinión pública ya estructurada, y de una creciente privatización de las universidades (incluidas las del Estado). Por tanto, su entrada a la industria fue muchas veces motivada por la rentabilidad económica asociada más que por el interés de generar conocimiento, toda vez que venden sus servicios a otras instituciones. En este grupo destacan la Dirección de Estudios Sociales (DESUC) de la Pontificia Universidad Católica, creada a comienzos de la década de 2000, y el Centro de Estudios de Opinión Ciudadana (CEOC) de la Universidad de Talca, nacido en 2003. Ambos organismos son creados por dichas universidades para prestar servicios a terceros vinculados con la medición de la opinión pública. Por otra parte, se destacan el Observatorio Político Electoral de la Universidad Diego Portales, creado en 2004, y el Centro de Estudios de Opinión Pública de la Universidad de Chile, cuyo ámbito de interés en relación con la opinión pública es exclusivamente político.

Finalmente, el último actor relevante de esta industria son los medios de comunicación, en especial los diarios El Mercurio y La Tercera, ambos ligados a grupos empresariales de derecha que apoyaron abiertamente la dictadura y que, desde 1999, comienzan a producir sus propias encuestas, cumpliendo así el doble rol de comunicadores y productores de encuestas de opinión. En efecto, a la función de "brújula" política que cumplen las encuestas de opinión se añade al mismo tiempo otra función de "oráculo", pues estas no solo indican "lo que quiere la gente" sino también lo que querrá en el futuro (Vommaro, 2008). En este contexto, las encuestas empiezan a ser "utilizadas como mecanismos de construcción de noticias y, por lo tanto, articulación de la agenda de debate público" (Cordero y Tapia, 2007, p. 21).

En resumen, es posible observar que actualmente en Chile conviven compitiendo dentro de la misma industria compañías internacionales dedicadas a los estudios de mercado y de opinión pública (Gallup, IPSOS, MORI, entre otras), con empresas nacionales (Adimark, Cadem, etc.), centros de estudios (CEP, CERC), fundaciones políticas y think tanks (Instituto Libertad y Desarro- 
llo, Fundación Futuro, Fundación Chile 21, etc.), universidades (UCH, UDP, PUC, UNAB, entre otras) y medios de comunicación de prensa escrita (El Mercurio, La Tercera).

El caso de Chile es expresivo de una realidad mundial. En efecto, en la actualidad la industria de la opinión pública no solo está consolidada, sino lo está en tanto fenómeno internacional y globalizado (Cordero y Tapia, 2007; Cordero, 2009).

\section{La noción de "dispositivo" y la reducción de la sociología}

El concepto "dispositivo" ha sido muy discutido por la filosofía política durante las últimas tres o cuatro décadas (Foucault, 1984, 2008 y 2014; Agamben, 2011; García Fanlo, 2011; Deleuze, 1990). Si bien su uso es extensivo e intensivo dentro de su obra, Foucault siempre se negó a entregar una definición por el subsecuente riesgo de cristalización. Sin embargo, en una entrevista de 1977 entrega elementos claves para entender a qué se refiere con este concepto:

Lo que trato de situar bajo ese nombre es, en primer lugar, un conjunto decididamente heterogéneo, que comprende discursos, instituciones, instalaciones arquitectónicas, decisiones reglamentarias, leyes, medidas administrativas, enunciados científicos, proposiciones filosóficas, morales, filantrópicas [...] En segundo lugar, [...] ese discurso puede aparecer bien como programa de una institución, bien por el contrario como un elemento que permite justificar y ocultar una práctica, darle acceso a un campo nuevo de racionalidad [...] En tercer lugar, por dispositivo entiendo una especie -digamosde formación que, en un momento histórico dado, tuvo como función mayor la de responder a una urgencia. El dispositivo tiene pues una posición estratégica dominante. (Foucault, 1984, pp. 128-129) 
Como se desprende de la cita, la noción de "dispositivo" en Foucault tiene relación con instituciones, discursos y prácticas vinculadas con una función veridictiva asociada a la detentación del poder, cuya finalidad está relacionada con la constitución de los sujetos (en tanto sujeción), pero que no aparece como evidente sino más bien como oculta.

En un nivel más analítico, tanto Agamben (2011) como García Fanlo (2011) coinciden en rescatar algunos aspectos principales o niveles de problematización de la noción de dispositivo en Foucault:

1. Es un conjunto heterogéneo de discursos, instituciones, instalaciones arquitectónicas, reglamentos, etc., tanto de lo dicho como de lo no dicho. El dispositivo es, entonces, la red que puede establecerse entre estos elementos.

2. El dispositivo es la naturaleza del vínculo entre estos elementos tan heterogéneos.

3. Siempre tiene una función estratégica concreta, la que a su vez se inscribe siempre en relaciones de poder.

4. Está vinculado con el acontecer, puesto que se entiende como una especie de formación que, en un momento histórico concreto, cumple la función de responder a una urgencia.

5. En consecuencia, el dispositivo es el resultado del cruce entre las relaciones de poder y de saber.

De esta lectura se desprende que, para el caso de este artículo, no se trata de utilizar la noción de dispositivo en referencia a la industria de opinión pública, sino más bien a la asociación entre sociología y opinión pública (tanto a la red que se establece entre estos elementos como a su naturaleza), que termina por reducir y homologar el quehacer disciplinario con la medición de opinión.

En efecto, como señala García Fanlo:

Un dispositivo no es algo abstracto. En tanto red de relaciones de saber/poder existe situado históricamente -espacial y temporalmente- y su emergencia siempre 
responde a un acontecimiento que es el que lo hace aparecer, de modo que para hacer inteligible un dispositivo resulta necesario establecer sus condiciones de aparición en tanto acontecimiento que modifica un campo previo de relaciones de poder. (2011, p. 2)

Entonces, para comprender este dispositivo que asocia sociología con opinión pública, es necesario llevar adelante una reconstrucción histórica del dispositivo (hacer una suerte de "genealogía", en palabras de Foucault), que permita evidenciar a qué emergencia responde y cómo y en qué medida modifica las relaciones de poder del campo en que se inserta, toda vez que el dispositivo no es solo una red entre instituciones ni la forma como se organizan internamente sus distintos componentes. Es más que eso. Para funcionar como dispositivo, dichas prácticas deben reconfigurar la naturaleza de las instituciones o campos que se asocian en la red.

Asimismo, siguiendo a Deleuze:

En todo dispositivo debemos desenmarañar y distinguir las líneas del pasado reciente y las líneas del futuro próximo, la parte del archivo y la parte de lo actual, la parte de la historia y la parte del acontecer, la parte de la analítica y la parte del diagnóstico. (1990, p. 160)

En consecuencia, a esta reconstrucción diacrónica e histórica para comprender el dispositivo se requiere sumar otra lectura más sincrónica, centrada en la descripción de las prácticas y los discursos que también lo sustentan. Este análisis toma en cuenta al menos tres dimensiones o componentes de todo dispositivo (Deleuze, 1990):

1. el régimen de luz: lo que se ve vs. lo que no se ve;

2. el régimen de enunciación: lo que se dice vs. lo que no se dice, $y$

3. la dimensión del poder: poder-saber, entendidos como líneas de fuerza que rectifican las curvas anteriores. 
Es posible evidenciar cómo lo señalado por Deleuze se vincula con el dispositivo mencionado, toda vez que cada dispositivo tiene su propio régimen de luz que distribuye lo visible y lo invisible; vale decir, hace "nacer o desaparecer el objeto que no existe sin ella" (1990, p. 155). Esta situación se observa en la creación de la opinión como producto respaldado científicamente por las encuestas sociológicas (Garrigou [2007] se refiere a la creación de un régimen de opinión). Se retomará en lo que sigue.

El último aspecto a destacar se relaciona con un rasgo muy expresivo de este dispositivo que homologa la sociología con la opinión pública, relacionado con que "a un discurso determinado el dispositivo le asigna un sujeto para que garantice su veracidad, prestigio y autoridad invistiéndolo de unos derechos adquiridos por competencia, saber, trayectoria, etc." (García Fanlo, 2011, p. 4). El caso del médico, analizado por Foucault en El nacimiento de la clínica (2008), es representativo de lo anterior, toda vez que su discurso está garantizado por aspectos institucionales en los cuales sus prácticas "son validadas como verdaderas y funcionan produciendo efectos de saber-poder que constituyen, al mismo tiempo, al sujeto enfermo al que atiende" (García Fanlo, 2011, p. 4).

Una situación similar se observa con los hacedores de encuestas quienes, a la luz de los medios de comunicación, se han ido transformando en expertos en todos los temas. En efecto, ya no se requieren expertos en religión, política, economía o ciencias para conocer e interpretar la realidad, basta con expertos hacedores de encuestas para conocer qué es lo que la opinión pública piensa de cada tema. En otras palabras, ya no se trata de los hechos, sino de opiniones que parecen ser siempre válidas solo por el hecho de estar cuantificadas.

\section{3. ¿Qué es la opinión pública?}

La preocupación y el debate en torno a la opinión pública no son exclusivos de la sociología ni son nuevos. Por el contrario, han suscitado la atención de destacados sociólogos, psicólogos 
sociales, filósofos e historiadores, y se discute si sus orígenes están en la Grecia y la Roma antiguas, en el siglo XVI con Maquiavelo (Bobillo, 1987), o es un fenómeno propio de sociedades liberales modernas (Heller, 1974). En consecuencia, hablar de “opinión pública" implica un problema, toda vez que no es posible arrimarse a una definición precisa (Cordero, 2009).

Con la finalidad de acercarse a una definición, en lo que sigue se abordan dos de las exposiciones más relevantes del siglo XX dentro de las ciencias sociales y la filosofía: las de Habermas y Bourdieu. Habermas se adentra en la reflexión sobre la opinión pública como parte de una preocupación filosófica mayor acerca del espacio público, y que incluiría también a su conocida "teoría de la acción comunicativa" (Boladeras, 2001). Para Habermas, la discusión pública es la única manera posible de superar los conflictos sociales, "gracias a la búsqueda de consensos que permitan el acuerdo y la cooperación a pesar de los disensos" (Boladeras, 2001, p. 53). En ese contexto, la opinión pública es una pieza clave para favorecer esta política deliberativa, pues aun cuando puede ser manipulada y deformada, constituye el eje de la cohesión social, así como de la construcción legítima de la política.

En este sentido, la opinión publica, nacida en el seno del espacio público, correspondería a un asunto ciudadano del "mundo de la vida", no a un asunto relativo a un determinado sistema político. A lo anterior agrega Habermas:

El ámbito de comunicación de las opiniones no públicas se contrapone a la esfera de circulación de una opinión quasi pública. Esas opiniones formales pueden reconducirse a instituciones tangibles; están oficial u oficiosamente autorizadas en calidad de comunicados, notificaciones, declaraciones, discursos, etc. De ahí que se trate primordialmente de opiniones que circulan, en un plano que escapa a la masa de la población. (1981, p. 271)

En definitiva, la opinión pública expresaría la racionalidad de la sociedad civil que, enfrentando el poder del Estado, entiende 
que dicha opinión no emana de principios abstractos, sino del ejercicio deliberativo de los ciudadanos privados, siendo inseparable de la discusión pública (Habermas, 1981).

Esta concepción habermasiana de la opinión pública es catalogada mayoritariamente como una propuesta normativa y basada en una concepción ilustrada, burguesa y liberal (Bouza, 2004). En ese contexto, no se adentra en la discusión más actual acerca del valor de las encuestas como medición de la opinión pública. Quien sí lo hace de manera directa y muy crítica es Bourdieu.

No hay mejor manera de reflejar la postura de Bourdieu que citando el título de una conferencia dictada en 1972: La opinión pública no existe (Bourdieu, 2000). Las razones que sustentan su afirmación son epistemológicas, políticas y metodológicas. A riesgo de la extensión, parece necesario exponer íntegramente los tres postulados que suponen implícitamente las encuestas de opinión pública:

[Primer postulado:] toda encuesta de opinión supone que todo el mundo puede tener una opinión; o, en otras palabras, que la producción de una opinión está al alcance de todos. Aun a riesgo de contrariar un sentimiento ingenuamente democrático, pondré en duda este primer postulado. Segundo postulado: se supone que todas las opiniones tienen el mismo peso. Pienso que se puede demostrar que no hay nada de esto y que el hecho de acumular opiniones que no tienen en absoluto la misma fuerza real lleva a producir artefactos desprovistos de sentido. Tercer postulado implícito: en el simple hecho de plantearle la misma pregunta a todo el mundo se halla implicada la hipótesis de que hay un consenso sobre los problemas, en otras palabras, que hay un acuerdo sobre las preguntas que vale la pena plantear. Estos tres postulados implican, me parece, toda una serie de distorsiones que se observan incluso cuando se cumplen todas las condiciones del rigor metodológico en la recogida y análisis de los datos. (Bourdieu, 2000, p. 220) 
Las razones para develar estos tres postulados están vinculadas entre sí. Así, por ejemplo, el supuesto consenso sobre los problemas acerca de los cuales vale la pena indagar sería, a juicio de Bourdieu, ilusorio, toda vez que las preguntas están subordinadas a intereses políticos coyunturales, lo que ejerce un enorme peso sobre la significación dada a las respuestas, así como también sobre la publicación de sus resultados. En efecto, las encuestas de opinión serían un instrumento de acción política que impone la ilusión de la existencia de la opinión pública como "sumatoria puramente aditiva de opiniones individuales"3, bajo una nueva ilusión de creer que, dado que todas las opiniones tienen el mismo peso, es posible obtener una suerte de opinión media por operaciones estadísticas.

De esta manera, a juicio de Bourdieu, los resultados de encuestas de opinión pública que aparecen en los diarios en formato de porcentajes, no serían más que

Un simple y puro artefacto cuya función es disimular que el estado de la opinión en un momento dado es un sistema de fuerzas, de tensiones, y que no hay nada más inadecuado para representar el estado de la opinión que un porcentaje. (Bourdieu, 2000, p. 221) ${ }^{4}$

Este análisis lleva a Bourdieu a concluir lo siguiente: "he aquí el efecto fundamental de la encuesta de opinión: constituir la idea de que existe una opinión pública unánime y, así, legitimar una política y reforzar las relaciones de fuerza que la sostienen o la ha-

\footnotetext{
3 Es interesante ver las similitudes de este debate con el que tuvo que dar la sociología en sus albores, durante el siglo XIX, en contra del contractualismo inglés y su afirmación irrestricta del individualismo metodológico y ontológico, y que se expresa de manera clara en el axioma durkheimiano de que la sociedad es más que la sumatoria de sus individuos (el todo es más que la suma de sus partes). Véase Durkheim (2000).

4 Este "encandilamiento numérico" tiene relación con que "decir las cosas con y a través de números añade a nuestros juicios y decisiones un sentido de rigor, experticia y universalidad de los cuales por sí mismos están desprovistos" (Cordero, 2009, p. 86).
} 
cen posible" (2000, p. 222). Porque lo cierto es que, aun cuando los gobiernos den cada vez más importancia a estas encuestas para definir el rumbo de sus propuestas políticas, es común que la opinión "ilustrada" o "informada" esté en contra de lo que señalan los sondeos de opinión. El ejemplo de lo que sucede actualmente con las Asociaciones de Fondos de Pensiones (AFP) en Chile es expresivo de esta situación, puesto que los sondeos indican que más del $80 \%$ de la población está a favor de reformar el sistema (Cadem, Estudio \#133), pero como esto choca con la opinión "informada", la respuesta gubernamental fue crear una comisión ad hoc. De esta manera, señala Bourdieu, "la comisión constituye una opinión pública informada que va a fijar la opinión informada como opinión legítima en nombre de la opinión pública que, además, dice lo contrario o no piensa nada" (2015, p. 55).

Por otra parte, y como ya se adelantó, estos postulados implícitos e ilusorios esconden que la finalidad de estas mediciones no es conocer la opinión pública sino más bien producirla, cuestión que -a juicio de Bourdieu- se lograría fundamentalmente a través de conminar a las personas a responder preguntas que no se habrían planteado por sí mismas. Este aspecto resulta crucial para evidenciar el funcionamiento del dispositivo en los términos antes señalados desde Foucault, porque, por un lado, permite hablar de "industria", toda vez que hay un producto a fabricar, y, por otro, por su función performativa, que no solo describe sino al mismo tiempo crea y modifica lo que busca conocer (Osborne y Rose, 1999; Cordero, 2009).

Finalmente, Bourdieu enfatiza que la medición de la opinión pública mediante encuestas se presenta como "una simple suma de opiniones individuales", cuando lo cierto es que

En las situaciones reales, las opiniones son fuerzas y las relaciones entre opiniones son conflictos de fuerza entre los grupos [...] La encuesta de opinión tradicional ignora al mismo tiempo los grupos de presión y las disposiciones virtuales que pueden no expresarse en forma 
de discurso explícito. Por ello es incapaz de generar la menor previsión razonable sobre lo que pasaría en situación de crisis. (Bourdieu, 2000, p. 231)

Estos son los principales fundamentos que llevan a Bourdieu a sostener que la opinión pública no existe. $\mathrm{O}$ al menos, existe solo como ficción.

Bourdieu no lucha contra fantasmas. Por el contrario, el significado y el uso que se da a las encuestas de opinión pública calza perfectamente con lo que describe, lo que se refleja bien en la definición aportada por Bouza (2004). Este autor sostiene que son dos los principales acercamientos conceptuales a la opinión pública: el concepto político y el sociológico. El concepto político de opinión pública se insertaría en la tradición clásica, desde la conceptualización francesa anterior y posterior a la Revolución de 1789 hasta Habermas, mientras que el sociológico nacería en 1937, con la publicación de la revista Public Opinion Quarterly de la Universidad de Oxford en Estados Unidos, y es básicamente la medición mediante el uso de encuestas. Bouza es abierto partidario de lo que llama "concepto sociológico", lo que implica hacerse cargo de las críticas que recibe desde la vertiente política.

En efecto, como defensa del concepto sociológico, Bouza aboga por la necesidad de un individualismo ontológico -aunque de manera implícita y algo ambigua-, toda vez que se debe "reivindicar a los individuos, porque ellos son la sustancia formante de toda Sociedad, Opinión o actividad humana, y son, por supuesto, un claro concepto empírico" (2004, p. 6). Dicho esto, sostiene que si bien no se sabe con claridad "qué es" la opinión pública, sí se sabe que "se le llama" opinión pública a lo que expresan numéricamente las encuestas. Vale decir, el concepto sociológico de opinión pública se definiría como el "agregado numérico de conductas verbales públicas en tanto que respuestas a preguntas en un cuestionario" (Bouza, 2004, p. 7). A esta definición, agrega:

Con esos datos trabajamos los sociólogos y desde esos datos vamos conociendo relativamente bien las conduc- 
tas sociales del hombre. Esto simplifica las cosas, aunque no conforme a nadie, y menos que nadie a los que definen los conceptos antes de observar a fondo las realidades que quieren representar con ellos, pero los que así hacen no hacen ciencia sino valores y normas, lo cual debe ser necesario, pero no puede impregnar de forma tan extrema el trabajo empírico. (2004, p. 7)

Resulta muy ilustrativa la cita anterior para ver cómo, desde esta concepción de la opinión pública, la sociología es por antonomasia la disciplina que debe hacerse cargo de su investigación, toda vez que sería algo así como la ruta de acceso privilegiada para conocer las conductas sociales humanas. Y lo hace, como se señala, "simplificando las cosas", lo que se expresa, por ejemplo, en que se ahorra debates profundos y de larga data al homologar opinión y conducta, lo que equivale a sostener que se conoce la realidad porque se conoce la opinión que los individuos tienen sobre ella. Esta justificación se debe a que la realidad habla por sí sola a través del trabajo empírico bien guiado metodológicamente (Bouza, 2004). De esta manera, para este tipo de acercamientos, anteponer conceptos -y aún más teorías- a esta realidad dada sería un ejercicio cuasiideológico vinculado con la axiología o la jurisprudencia, pero no con la ciencia.

Con todo, lo que interesa destacar es que el dispositivo que homologa "medición de la opinión pública" con "sociología" no solo debe desmontarse mediante la crítica a las encuestas de opinión, sino también -y muy especialmente- a la idea de que la cientificidad del trabajo sociológico se sustentaría en la aplicación aséptica de procedimientos metodológicos neutros, obviando la ontología que le subyace. Más bien, como señala Archer (2009), no es concebible una aplicación metodológica neutra desprovista de ontología. Por el contrario, "lo que se asume como realidad social no puede sino influenciar el cómo se la estudia [...] siempre hay una conexión entre la ontología social y la metodología 
explicativa (sin importar cuán encubierta o poco útil sea)" (2009, p. 44$)^{5}$.

\section{Las falencias (y falacias) del dispositivo}

Podrían resumirse en tres los principales argumentos de legitimación de la medición de opinión pública más difundidos y exitosos: 1) que corresponde a un ejercicio científico, lo que le entrega validez técnica, 2) que implica una práctica democrática y que, al mismo tiempo, está a su servicio, y 3) que sus resultados son el reflejo de verdades. Estos argumentos están necesariamente imbricados. A continuación, se realiza un breve análisis crítico de cada uno en tanto falencias del dispositivo -devenidos en falacias- con el fin de desmontarlos.

\subsection{De su carácter científico}

La idea de que la medición de la opinión pública mediante encuestas corresponde a un ejercicio científico no es espontánea. Por el contrario, es producto de un largo y sostenido trabajo realizado por los hacedores de encuestas. Resulta ilustrativa de esta construcción la anécdota de Gallup, padre fundador y pionero a nivel mundial de la industria de encuestas de opinión, quien firmaba todos sus documentos utilizando el título de doctor (Garrigou, 2007). Esto redunda en que las encuestas no son cualquier producto, son información fiable. Y su fiabilidad tendría como base la aplicación de criterios metodológicos científicos. Vale decir, designan la técnica como criterio de cientificidad. Con esto obvian no solo el compromiso ontológico que hay tras ella (Archer, 2009), sino también olvidan que la metodología es únicamente un aspecto del proceso científico, toda vez que no basta con llevar a cabo una medición -aun cuando ésta sea exacta- para hacer ciencia. Como señala Garrigou:

En la sección final se retoma con más detalle este planteamiento de Archer. 
Las cuestiones técnicas de representatividad de las poblaciones, márgenes de error, métodos de cuotas o aleatorios, correcciones o ponderaciones, etc., se convierten en falsos temas si se las aísla y se olvida preguntar por lo que miden las encuestas. No basta contar para hacer ciencia. (2007, p. 18)

Otra característica de esta pretendida cientificidad es que permite rechazar las críticas invocando ignorancia e incompetencia técnica y científica de quienes las profieren. Como señala Méchet: "los que critican las encuestas son los que menos conocen las técnicas" (Garrigou, 2007, p. 16). Así, la alta sofisticación metodológica de sus técnicas sería una suerte de garantía científica ante posibles errores, pero sobre todo ante la crítica del lego.

\subsection{De su carácter democrático}

En cuanto a la supuesta condición democrática de los sondeos de opinión, es posible diferenciar dos planos. Por un lado, "darían voz a los sin voz", toda vez que permitirían la expresión libre, no coaccionada, gratuita y publicitada de su opinión al ciudadano común ${ }^{6}$; por otro está la idea de que las mediciones de opinión pública solo existen en países democráticos -aparecen en países de la ex URSS y en América Latina junto con los procesos de democratización ${ }^{7}-$, puesto que solo allí los gobernantes estarían interesados en conocer el parecer de sus gobernados. "En suma, atacar a las encuestas (de opinión) sería atacar a la democracia" (Garrigou, 2007, p. 15).

Pero lo cierto es que hay un sinnúmero de prácticas que son legales solo en los países considerados democráticos, sin que por

\footnotetext{
6 La última tendencia adoptada por los partidos políticos en Chile -y parte del mundo- para definir a sus candidatos por medio de encuestas de opinión previas a la elección, expresa en forma concentrada este punto.

7 El caso de toda América Latina resulta muy expresivo de esta tesis, puesto que, luego de un oscurantismo durante las dictaduras, las encuestas de medición de la opinión pública se retoman con fuerza tras sus caídas (Aguiar, 2009).
} 
ello se las defina como democráticas, como la pornografía (Garrigou, 2007). Asimismo, como se señaló con Bourdieu, el interés de los gobernantes por el parecer de los gobernados queda en entredicho cada vez que esa opinión toca intereses económicos poderosos o demanda cuestiones estructurales.

\subsection{De su carácter de verdad}

Por último está la idea de que las encuestas de medición de la opinión pública son el reflejo mensurado de verdades. En efecto, las encuestas funcionan con el supuesto implícito -ya señalado por Bourdieu-de que basta con preguntar a los individuos lo que piensan y/o hacen para efectivamente saberlo. "Los encuestadores serían así los primeros en obtener directamente la verdad, y su privilegio sería tanto más exorbitante porque la obtendrían casi siempre de encuestados ignorantes de sus propias razones" (Garrigou, 2007, p. 33).

Pero resulta evidente, y hasta fundante para disciplinas como la sociología y la psicología, que el trabajo científico debe develar lo que no es aparente, lo que aparece opaco a los individuos y a sus conciencias. De lo contrario, si bastara con preguntarle a las personas para conocer sus más complejos sistemas de intereses, deseos, creencias, aptitudes, etc., y además, si luego bastara con sumar esas respuestas para saber lo que sucede a nivel colectivo, las ciencias sociales serían inútiles. En efecto, en línea con la idea de "régimen de luz" expuesta con Deleuze (1990), Garrigou (2007) sostiene que esta concepción de la opinión pública genera un régimen de opinión, que toma por verdad lo que los individuos dicen sin que exista un cuestionamiento epistemológico de si ésta es la mejor manera de acceder a la verdad del pensamiento. En efecto:

En el régimen de opinión constituido por las encuestas, se invierte el orden comprehensivo; en lugar de acceder a la conciencia por las prácticas, se pretende acceder a las prácticas por la conciencia. Es una regresión intelec- 
tual cuya amplitud parece no haber sido pensada a fondo. (Garrigou, 2007, p. 34)

Finalmente, este régimen de opinión se sustenta en un compromiso ontológico que desemboca en un constructivismo radical, que piensa la realidad como constituida básicamente por discursos y opiniones. En efecto, esta posición aparentemente inocua de tomar por verdad lo que los individuos dicen, asumiendo que sus pensamientos serán guía inquebrantable de su acción, corre el riesgo de intentar conocer y manejar diversos temas conflictivos dentro de la sociedad -como la homofobia por ejemplo- a través de sondeos de opinión.

\section{La falacia de la neutralidad metodológica: ejemplos}

La idea de neutralidad metodológica es el corolario tras la pretensión de comprender las encuestas de medición de opinión pública como científicas, democráticas y verdaderas. Y es que parte importante de su atractivo político y comercial se basa en el supuesto de que recogen la realidad sin mayores intervenciones teóricas (Bouza, 2004).

Ahora bien, como señala Bobillo (1987), contra la opinión pública crítica se desarrolla una opinión pública manipulada, incluso deformada. Porque las encuestas de opinión están provistas de un carácter performativo que las constituye dualmente como termómetro o brújula y, al mismo tiempo, como oráculo (Cordero y Tapia, 2007; Vommaro, 2008). Con esa finalidad, en este apartado se presentan algunos ejemplos de encuestas de medición de opinión pública en las que es posible observar la falacia de la neutralidad metodológica ${ }^{8}$. Se presentan tres tipos de falacia de neutra-

\footnotetext{
8 Este ejercicio no pretende ser un estudio sistemático de dicha práctica ni tampoco de las instituciones que las sustentan, sino busca dar un apoyo empírico al desarrollo teórico-conceptual expuesto.
} 
lidad metodológica, apoyados por ejemplos actuales extraídos de encuestas de opinión nacionales e internacionales.

\subsection{Influencia en los resultados}

El primer tipo de falacia es el más evidente y se vincula, por un lado, con cuestionarios que inducen cierto tipo de respuestas, $\mathrm{y}$, por otro, con los análisis de resultados que son generados $-\mathrm{y}$ difundidos a través de los medios de comunicación- por las mismas instituciones que aplicaron la encuesta, y en los que se establecen relaciones de causalidad sin tener evidencia suficiente. En ambos casos, la falacia de neutralidad metodológica se expresa en que los intereses de quienes ejecutan o mandatan la medición influyen de manera evidente en los resultados.

El primer caso de preguntas que inducen cierto tipo de respuestas se puede ejemplificar con las encuestas formuladas por las dos principales empresas de medición de opinión pública en Chile. Así, las encuestas "Evaluación del gobierno", de Adimark, y "Plaza Pública", de Cadem, ambas de 2016, realizan la misma pregunta9: “¿Usted está de acuerdo o en desacuerdo con la reforma a la educación del gobierno?". Aquí llama la atención la generalidad de la pregunta, toda vez que la reforma a la educación del gobierno -si es que puede englobarse todo en una sola reformaconsta de varias reformas diferentes (parvularia, superior, ley de inclusión, carrera docente, desmunicipalización), y cada una de ellas contiene un sinnúmero de propuestas o modificaciones que apuntan a diferentes objetivos.

Lo mismo sucede con las preguntas que ambas empresas hacen sobre las reformas tributaria y laboral, o sobre la aprobación

\footnotetext{
9 En la encuesta "Plaza Pública" \#184 de Cadem (semana del 24 de julio de 2017) se sigue haciendo la misma pregunta. No así en la "Evaluación del gobierno" de Adimark. Para efectos de comparación se toma la encuesta "Plaza Pública" \#134 de Cadem, correspondiente a la primera semana de agosto de 2016, y la "Evaluación del gobierno" de julio de 2016 de Adimark.
} 
del gabinete. Al no distinguirse claramente qué se está preguntando y al haber solo una opción dicotómica de respuesta (acuerdo/ desacuerdo, aprueba/desaprueba), la pregunta se hace confusa por generalidad y por limitación de opciones, lo que no identifica las tensiones del fenómeno ni permite análisis más fértiles.

Otro ejemplo lo entrega la encuesta \#130 de "Plaza Pública" Cadem, correspondiente a la semana del 11 de julio de 2016, en la que se intenta profundizar en la opinión sobre la reforma educacional del gobierno a través de preguntas específicas. Una de ellas señala lo siguiente: “ ¿Ud. cree que la educación universitaria debe ser gratuita para...?", entregando tres opciones: 1) "el $50 \%$ de los estudiantes más vulnerables", 2) "El 70\% de los estudiantes más vulnerables y de clase media", y 3) "El 100\% de los alumnos, incluyendo a las familias de más altos ingresos". No creo necesario ahondar en la evidente influencia en la respuesta que tienen expresiones calificativas (sin justificación metodológica) como "vulnerables". Pero lo que resulta más notorio es el cambio de sujeto sobre el que versa la pregunta (en las dos primeras opciones es el estudiante, mientras que en la última se pasa injustificadamente a la familia). Todo indica que Bourdieu, Chamboredon y Passeron tienen razón cuando señalan que "en tanto no hay registro perfectamente neutral no existe una pregunta neutral [...] [No reconocerlo] es estar expuesto evidentemente a recoger un flatus vocis que no tiene otra significación que la que le confiere el sociólogo tratándolos como un discurso significante" (2002, p. 63).

Por otro lado, en relación con la influencia del análisis en el establecimiento de relaciones de causalidad, el caso de la encuesta mensual de Adimark es claro. El documento comienza con el análisis de las respuestas, vinculándolas con las noticias del mes que influyeron en ellas. Además de que la selección de noticias influyentes ya es un establecimiento de causalidad espuria ( $\sin$ mencionar la influencia de los dueños de los medios de generación de noticias), es muy indicativo que siempre los resultados se entreguen con una conferencia de prensa, en la que hablan los 
expertos en encuestas explicando el porqué de los resultados, con causalidades notables, como la indicada por su director, Roberto Méndez, a propósito de los resultados de aprobación de Bachelet en abril de 2016: "la muerte de Aylwin favoreció a la presidenta. Hizo revivir una actitud más favorable para la política" (ADNRadio, abril 2016).

Lo anterior no implica, por supuesto, que los intereses siempre sean explícitos y se manipulen sin miramientos todas las mediciones. No. Implica que todo sujeto -incluyendo a quienes hacen las encuestas- es portador de intereses y contradicciones, los cuales influyen indefectiblemente en su quehacer. Además, estos intereses no se restringen a los políticos; la ideología también se relaciona fuertemente con intereses de clase, de género, profesionales, o simplemente personales, inclusive.

\subsection{Influencia sobre la agenda pública}

La segunda falacia metodológica se relaciona con la creación y realización de encuestas dirigidas a influir en la agenda política. Esta falacia se basa en la pretensión de construir una realidad a partir de influir en la sensación colectiva de que ésta existe, confundiendo así la realidad con la opinión que determinados individuos tienen sobre ella, cuestión que vuelve más manipulable políticamente la medición de opinión pública por la mencionada performatividad (Cordero, 2009; Osborne y Rose, 1999; Porter, 1995).

Ejemplos de lo anterior se observan en la encuesta "Plaza Pública" de Cadem, en la que se pregunta semanalmente: “¿Ud. cree que en el momento actual la economía chilena está: progresando, estancada o en retroceso?". Llama la atención que en la medición de julio de 2017 -última medición al momento de escribir este artículo- el 83\% crea que está estancada o retrocediendo (Plaza Pública Cadem \#184), cuando lo cierto es que, de acuerdo con los parámetros establecidos, al momento de la medición la economía chilena estaba creciendo 1,3\% (IMACEC de mayo de 2017). 
¿Buscan dichas mediciones conocer qué piensa la opinión pública sobre la economía, o bien aportar a una sensación de recesión económica? Lo que sin duda complejiza y vuelve más atractivo su uso desde la política formal, es que en fenómenos sociales que se constituyen desde un fuerte componente de percepción -como la economía- existe la posibilidad de que se produzca el efecto de la profecía autocumplida, que Merton define como "una definición 'falsa' de la situación que despierta un nuevo comportamiento que hace que la falsa concepción original de la situación se vuelva 'verdadera'”' (1964, p. 421).

Otro ejemplo, aún más claro que el anterior, lo representa la muy difundida medición de victimización, inseguridad y percepción de la delincuencia, que en Chile está a cargo de una asociación entre la Fundación Paz Ciudadana y Adimark. Lo notable de este ejemplo es la creación de un artefacto llamado "percepción de la delincuencia", que ha alcanzado mucha difusión y popularidad en América Latina y Chile, toda vez que tiene relación con la sensación de inseguridad de los individuos y no con los datos reales de delincuencia. Claramente, es mucho más fácil obtener datos de la sensación de inseguridad que de los hechos delictivos mismos, pero el hecho de que estas encuestas tengan tal influencia en la generación de políticas públicas y en su difusión en los medios de comunicación, se explica principalmente por lo mismo que el ejemplo anterior: por la posibilidad de crear determinada realidad o, a lo menos, influir en ella. A esta situación -que no es exclusiva de Chile- Dammert (2009) la llama creación de "falsas alarmas", cuya finalidad no es solo distorsionar parte de la realidad, permeando el debate público con una exagerada sensación de inseguridad, sino sobre todo influir en el diseño de políticas públicas para el control y prevención del crimen.

\subsection{La opinión como verdad (régimen de opinión)}

Finalmente, el último tipo de falacia de neutralidad metodológica dice relación con lo que Garrigou (2007) llamó "régimen de 
opinión", que consiste en creer que la opinión constituye verdad. Esto sin duda acarrea el peligro de la desinformación y la manipulación.

Un ejemplo muy claro de este tipo de falacia se puede observar en dos encuestas realizadas por la empresa Gallup. La primera se inscribe en el marco de la encuesta anual sobre medioambiente, aplicada en Estados Unidos, y entre sus resultados para el año 2016 arrojó que "el 65\% considera que las alzas en la temperatura de la Tierra durante el último siglo son atribuibles principalmente a las actividades humanas en lugar de causas naturales" (La Tercera, 22/03/2016). Asimismo, "el 59\% dice que hoy los efectos del calentamiento global ya han comenzado, por encima del 55\% de marzo de 2015" (La Tercera, 22/03/2016).

¿Es posible un ejemplo más claro de la falacia de constitución de la opinión en verdad? Nada importa que el calentamiento global sea uno de los grandes problemas de discusión científica del momento, ni los miles de estudios que tratan de argumentar y demostrar con evidencias los alcances de este fenómeno. No. Lo que interesa en un régimen de opinión es si la población lo cree o no. Por supuesto, no se necesita ser experto en ciencias geológicas o medioambientales para tener una idea de los intereses políticos y económicos que hay tras estas mediciones.

El segundo ejemplo tiene relación con una nota publicada por el diario La Tercera el 25 de junio de 2015, titulada "Chile entre los seis países con mayor bienestar del mundo", que se inscribe en el contexto de una encuesta internacional realizada por la empresa Gallup, la cual ranqueó a 145 países en función de su índice de bienestar, medido por cinco puntos, en el que Chile aparece en el sexto lugar. ¿Qué se está midiendo realmente? Ciertamente el bienestar es en sí mismo un concepto impreciso, que apela a estados emocionales individuales, pero es evidente además la intención de ver que la única verdad posible es la subjetividad, y que ésta se mide a través de encuestas de opinión. $O$ bien que la única objetividad posible es la sumatoria de opiniones. 


\section{Las carencias del dispositivo}

En el interés de desmontar el dispositivo que homologa la práctica sociológica con la medición de la opinión pública, propósito que ha mostrado hasta aquí las falencias del mismo, es posible sumar también ciertas carencias que tienen relación con el hecho de que la sociología es una disciplina intrínsecamente explicativa y, como tal, es irreductible a las encuestas de opinión y a método alguno. Esta idea se desarrolla fundamentalmente desde las propuestas de Archer (2009).

\subsection{La medición de opinión pública como conflación ascendente}

Archer plantea que el dilema de la relación entre individuo y sociedad (agencia y estructura) ha sido, es y será el problema sociológico fundamental. Ahora bien, no se trata de un problema con solución posible, toda vez que se deriva de lo que la sociedad intrínsecamente es. Pero la dificultad mayor viene dada porque la teoría social ha estado históricamente dominada por teorizaciones unidimensionales para abordar este problema, que Archer reúne en tres tipos de conflación ${ }^{10}$ : ascendente, descendente y central.

No viene al caso explicar en detalle acá la teoría de Archer ni los tres tipos de conflación, pero sí resulta atingente definir la conflación ascendente, toda vez que la entiende como aquella teorización que concibe las estructuras sociales como el resultado de la agregación de la acción individual y, por tanto, su exponente paradigmático sería el individualismo metodológico y ontológico, que entiende al individuo como el elemento principal -sino exclusivo- para la comprensión de la realidad social.

\footnotetext{
10 El término "conflación" viene del original en inglés (conflation), neologismo utilizado por la autora y que en otras publicaciones ha sido traducido como "fusión", y se refiere al ejercicio teórico-conceptual por el cual uno o ambos elementos en juego (individuo y sociedad) se funde en el otro, perdiendo su existencia propia.
} 
Las encuestas de medición de opinión pública serían entonces un claro ejemplo de conflación ascendente, en tanto comprenden los fenómenos macrosociales como resultado de acciones individuales agregadas, lo que se apoya en el individualismo metodológico y ontológico expresado ilustrativamente en la defensa de Bouza (2004) antes expuesta. El supuesto básico de este tipo de conflación es una premisa homológica: es posible llegar a la explicación del sistema social mediante un proceso de acumulación de acciones individuales, toda vez que "se encuentran las mismas propiedades (ni más, ni menos, ni diferentes) a lo largo y ancho de la sociedad" (Archer, 2009, p. 37).

$\mathrm{Al}$ asumir un conflacionismo ascendente -aunque de manera no explícita, lo que implica otro problema que se abordará luego-, las encuestas de opinión pública niegan autonomía y existencia real a fenómenos estructurales o colectivos que están más allá de los individuos, lo que implica entenderlos como no causales (epifenoménicos), dado que solo el individuo y su agregación tendrían la posibilidad de generar acciones con consecuencias reales.

De esta manera, para quienes defienden este tipo de conflación -como los hacedores de encuestas de opinión pública- la sociología debe remitirse a aquello que es observable, es decir, a los individuos, toda vez que serían la única garantía de realidad por sobre "fantasmas metafísicos", como las clases sociales, las mujeres, la sociedad, etc. "Al asumirlo como elemento último, el individuo del individualismo es considerado él mismo también como inmune a cualquier reducción (posterior) y a la vez todas las cosas sociales pueden ser reducidas a él" (Archer, 2009, p. 74).

Pero las consecuencias - a veces inadvertidas- de lo anterior tienen relación con que, a juicio de Archer:

Lo que se asume que existe debe influenciar las consideraciones sobre cómo debe ser explicado [...] [De esta manera,] la ontología social que se acepta juega un poderoso 
rol regulativo en relación con la metodología explicativa, por la razón básica de que conceptualiza la realidad social en ciertos términos y de ese modo identifica qué hay que explicar y también descarta explicaciones en términos de entidades o propiedades que se asumen como no existentes. (2009, pp. 48-49)

[En efecto,] lo que se asume como realidad social no puede sino influenciar el cómo se la estudia [...] siempre hay una conexión entre la ontología social y la metodología explicativa (sin importar cuán encubierta o poco útil sea). (2009, p. 44)

Por lo tanto, si la medición de la opinión pública pretende ser científica, en primer lugar debe asumir su posición ontológica y la conexión que ésta tiene con sus métodos y resultados.

\subsection{La sociología como práctica científica explicativa de la realidad social}

El problema central para las ciencias sociales es la inevitable y siempre problemática relación entre lo epistémico y lo ontológico (Venables, 2016). En efecto, en tanto aspecto epistémico, la realidad social requiere ser creída para poder existir (el dinero, una fiesta, la familia, el Estado e incluso este artículo, son lo que son en tanto así lo creemos); en otras palabras, es dependiente de los estados mentales en juego (Searle, 1997), situación que no se observa en la naturaleza, toda vez que ésta existe con independencia de los seres humanos y sus estados mentales.

Pero esta condición necesaria no es suficiente. Vale decir, no porque la realidad social deba ser creída para poder existir significa que se constituye únicamente por esta creencia. En efecto, la creencia no es el fundamento de su existencia, sino uno de sus componentes. Su fundamento está en la actividad práctica, en la acción (Venables, 2016). O, como señala Bourdieu a propósito de la diferencia entre doxa e ideología, "el mundo social no funcio- 
na en términos de conciencia, funciona en términos de prácticas, mecanismos, etc." (Zizek, 2003, p. 298).

Por lo tanto, es necesario comprender que la inevitable relación epistémico-ontológica de lo social se da en un contexto de realidad que es independiente de los estados mentales en juego. Como señala Bhaskar:

La sociedad antecede al individuo. Quien va a la iglesia o habla un lenguaje encuentra sus creencias o lenguaje ya formados al nacer, por lo que las personas no crean la sociedad [...] Las antecede la estructura social [que] está siempre ya formada. (Archer, 2009, p.198)

En consecuencia, el componente ontológico de la realidad social no puede ser obviado, toda vez que es tan constitutivo de la realidad social como el epistémico. Así, la sociología basa su especificidad y cientificidad en su capacidad de hacer frente a esta condición epistémico-ontológica de la realidad social. Por lo tanto, cuando las encuestas de opinión pública intentan establecer la verdad y hacer ciencia social a través de la medición de las creencias y opiniones de los individuos, no están considerando los constreñimientos, sanciones o restricciones sobre ellos, así como tampoco el aspecto material expresado en acciones o prácticas que dan su especificidad a la realidad social. En definitiva, no están haciendo sociología.

La propuesta de Archer al respecto -que de paso busca evitar el conflacionismo ascendente- está en reconocer la existencia de propiedades emergentes estructurales (PEE) que son irreducibles a los individuos, puesto que éstos no las identifican en su interacción social, toda vez que ésta solo produce una apreciación parcial y falible del contexto estructural en el que los individuos están inmersos. En esta misma línea, Bourdieu et al. sostienen que:

El hecho científico se conquista, construye y comprueba [...] No es sólo interrogarse sobre la eficacia y el rigor formal de las teorías y de los métodos, es examinar a las 
teorías y los métodos en su aplicación para determinar qué hacen con los objetos y qué objetos hacen. (2002, p.25) (cursivas mías)

En consecuencia, debe rechazarse todo empirismo que reduzca el acto científico a la comprobación.

En definitiva, la sociología no se entiende sino como una práctica científica al servicio de la explicación de la realidad social; tarea compleja que requiere un trabajo teórico y práctico que no se agota en la prueba experimental, toda vez que ésta es ciega e inconducente si no se acompaña de una teoría (Archer, 2009; Bourdieu et al., 2002). Solo así es posible un conocimiento sociológico que, primero, rompa con el saber inmediato, luego construya su objeto de estudio y, finalmente, pueda comprobarlo. El dispositivo que reduce y homologa la práctica sociológica a la medición de la opinión pública por medio de encuestas no opera de esta forma, por lo que no logra constituirse en una práctica científica ni sociológica.

\section{A modo de conclusión}

El propósito central de este artículo fue llevar adelante un ejercicio de análisis que se constituyera en un aporte para la tarea de desmontar un dispositivo relativamente extendido, que reduce y homologa la disciplina sociológica a la medición de la opinión pública a través de encuestas, y que resulta nocivo para la práctica sociológica en particular y para las posibilidades de transformación de la sociedad en general.

En un momento en el que el uso de las encuestas se ha difundido rápidamente sin una reflexión concordante, especialmente en América Latina (Angell, 2009; Cordero, 2009), este artículo busca aportar en una dirección analítica y crítica acerca del uso que de ellas se hace, de cómo reducen el espectro de acción de la sociología y de cómo influyen en la percepción que nos hacemos de las sociedades en que vivimos. 
Ahora bien, es importante señalar que la crítica está dirigida a las encuestas de opinión y no a las encuestas en general, y mucho menos a la utilización de métodos cuantitativos en ciencias sociales. Estos, por el contrario, tienen bien merecido un lugar destacado dentro de la investigación social. Más aún, en estricto rigor la crítica tampoco está dirigida a las encuestas de opinión per se, sino más bien a la idea que identifica la opinión pública con los resultados de dichas encuestas, al mismo tiempo que sustenta esa identificación en el carácter pretendidamente científico (sociológico) que ellas tendrían.

Asimismo, este artículo no pretende hacerse cargo de una crítica política ni de una historización crítica sistemática vinculada con las encuestas de medición de la opinión pública en Chile. Para ello se requiere, a lo menos, una investigación acabada acerca de los controladores de la industria y de los intereses políticos, económicos y personales en juego. Este artículo pretende, más bien, realizar una crítica epistemológica, ontológica y metodológica que, desmontando el dispositivo, contribuya a reivindicar el carácter eminentemente explicativo, crítico y transformador de la sociología.

Hechas las críticas y expandido el concepto de "sociología", se logra desmontar el dispositivo, dando cuenta de que la medición de la opinión pública a través de encuestas, si bien puede formar parte del quehacer sociológico -y de hecho lo hace-, no es suficiente en sí misma como para calificarla de práctica sociológica, y, más importante, no cuenta con las características explicativas propias de las ciencias sociales como para que la sociología se vea reducida a ella.

\section{Referencias}

Agamben, G. (2011). ¿Qué es un dispositivo? Revista Sociológica, 26(73), 249-264.

Aguiar, C. (2009). Apuntes para la historia de los estudios de opinión pública en América Latina. En: R. Cordero (editor), La socie- 
dad de la opinión. Reflexiones sobre encuestas y cambio político en democracia. Santiago: Ediciones Universidad Diego Portales.

Angell, A. (2009). Prefacio. En: R. Cordero, R. (editor), La sociedad de la opinión. Reflexiones sobre encuestas y cambio político en democracia. Santiago: Ediciones Universidad Diego Portales.

Archer, M. (2009). Teoría social realista. El enfoque morfogenético. Santiago: Ediciones Universidad Alberto Hurtado

Bobillo, F. (1987). La opinión pública [Versión electrónica]. Revista de Estudios Políticos, 58, 37-60.

Boladeras, M. (2001). La opinión pública en Habermas [Versión electrónica]. Revista Anàlisi, 26, 51-70.

Bourdieu, P. (2000). Cuestiones de Sociología. España: Istmo.

Bourdieu, P. (2002). Razones prácticas. Barcelona: Editorial Anagrama.

Bourdieu, P. (2015). Sobre el Estado: Cursos en el College de France (19891992). Barcelona: Editorial Anagrama.

Bourdieu, P., Chamboredon, J. C. y Passeron, J. C. (2002). El oficio de sociólogo. Presupuestos epistemológicos. Argentina: Siglo XXI editores.

Bouza, F. (2004). Desventuras de un concepto claro y distinto: Opinión pública. En: VV. AA. Reflexiones sociológicas. Libro homenaje a José Castillo Castillo. España: Centro de Investigaciones Sociológicas.

Cordero, R. (Editor). (2009). La sociedad de la opinión. Reflexiones sobre encuestas y cambio político en democracia. Santiago: Ediciones Universidad Diego Portales.

Cordero, R. y Tapia, G. (2007). Sumando opiniones: antecedentes históricos y desarrollos metodológicos de la industria de la opinión pública en Chile. Documentos de trabajo ICSO. Recuperado el 8 de noviembre de 2017, de http:/ / www.icso.cl/ images/Paperss/cuartopaper.pdf

Dammert, L. (2009). ¿Falsa alarma? Temor, crimen y opinión pública en Chile. En: R. Cordero (Editor), La sociedad de la opinión. Reflexiones sobre encuestas y cambio político en democracia. Santiago: Ediciones Universidad Diego Portales.

Deleuze, G. (1990). ¿Qué es un dispositivo? En: VV. AA. Michel Foucault, filósofo. España: Editorial Gedisa. 
Durkheim, E. (2000). Las reglas del método sociológico y otros escritos. España: Alianza Editorial.

Foucault, M. (1984). Saber y verdad. Madrid: Ediciones de la Piqueta.

Foucault, M. (2008). El nacimiento de la clínica. Buenos Aires: Siglo XXI editores.

Foucault, M. (2014). Vigilar y castigar. Nacimiento de la prisión. México: Siglo XXI editores.

García Fanlo, L. (2011). ¿Qué es un dispositivo: Foucault, Deleuze, Agamben? A parte Rei. Revista de Filosofía. Recuperado el 8 de noviembre de 2017, de http://serbal.pntic.mec.es/ AParteRei/fanlo74.pdf

Garrigou, A. (2007). La embriaguez de las encuestas. Santiago: Editorial LOM.

Habermas, J. (1981). Historia y crítica de la opinión pública. La transformación estructural de la vida pública. Barcelona: G. Gili.

Heller, H. (1974). Teoría del Estado. México: FCE.

Marx, K. (1973). El Capital, Vol. 1. México: FCE.

Merton, R. (1964). Teoría y estructuras sociales. España: FCE.

Osborne, T. y Rose, N. (1999). Do the Social Sciences Create Phenomena? The Example of Public Opinion Research [Electronic version]. British Journal of Sociology, 50(3), 367-396.

Porter, T. (1995). Trust in Numbers. The Pursuit of Objectivity in Science and Public Life. Princeton: Princeton Universitiy Press.

Searle, J. (1997). La construcción de la realidad social. Barcelona: Paidós.

Venables, J. P. (2016). Aportes para una ontología social realista. Revista Cinta de Moebio. Recuperado el 8 de noviembre de 2017, de http://www.moebio.uchile.cl/56/venables.html

Vommaro, G. (2008). Lo que quiere la gente. Los sondeos de opinión y el espacio de la comunicación política en Argentina (1983-1999). Argentina: Prometeo Libros.

Zizek, S. (comp.). (2003). Ideología: un mapa de la cuestión. España: FCE.

\section{Documentos electrónicos y artículos periodísticos:}

Adimark. (2016). Evaluación del gobierno de julio. Recuperado de http:/ / www.adimark.cl/es / estudios / documentos / 29 eval\%20gobierno\%20jul_2016.pdf 
Cadem. (2017). Encuesta Plaza Pública \#130, 134 y 184. Recuperado de http:/ / plazapublica.cl/temas/encuestas/

Espinosa, C. (2015). Chile está entre los seis países con mayor bienestar del mundo. La Tercera, Tendencias. Recuperado de http:/ / www.latercera.com/noticia/tendencias/2015/06/659636092-9-chile-entre-los-seis-paises-con-mayor-bienestar-del-mundo.shtml

Méndez, R. (4 de mayo de 2016). Roberto Méndez y la Adimark: “La muerte de Aylwin favoreció a la Presidenta". ADN Radio. Recuperado de http:/ / www.adnradio.cl/noticias/politi$\mathrm{ca} /$ roberto-mendez-y-la-adimark-la-muerte-de-aylwinfavorecio-a-la-presidenta/20160504/nota/3124354.aspx

Sepúlveda P. (2016). Crece población en EE.UU. que cree en el cambio climático. La Tercera, Tendencias. Recuperado de http:// www.latercera.com/noticia/tendencias/2016/03/659673322-9-crece-poblacion-en-eeuu-que-cree-en-el-cambio-climatico.shtml 